\title{
LOUIS PASTEUR AND MOLECULAR MEDICINE: A CENTENNIAL CELEBRATION
}

\author{
Maxime Schwartz, Ph.D., Director General, Institut Pasteur
}

Unesco and the Institut Pasteur have designated 1995 "The Year of Louis Pasteur" throughout the world; today, 100 years after Pasteur's death, we can step back and fully appreciate the impact of his body of work. Although Pasteur is perhaps best known for his triumphs in the field of infectious diseases, his discoveries have had relevance to a broad spectrum of fields in clinical research. Pasteur laid the foundations for research into areas ranging from the interactions among all biological molecules, to the molecular nature of infectious diseases, to the development of vaccines. The recently established discipline of molecular medicine celebrates Pasteur as one of the earliest and greatest of its founders.

The image that comes most readily to mind from Pasteur's early period is that of the young 26-year-old chemist, fresh out of the Ecole Normale, sorting out crystals of tartaric acid, while the eminent crystallographer, Jean-Baptiste Biot, looked on in astonishment. After the experiment was over, Pasteur himself recalled that the illustrious old man was deeply moved. "My dear boy," he said taking Pasteur by the arm, "I have loved science so much all my life that this touches my heart."

What was this dramatic discovery? Pasteur had demonstrated to Biot that a rare form of tartrate, known as paratartrate, was in fact composed of equal quantities of two types of molecules whose crystals, although very similar, could be distinguished by the orientation of one minute facet. These two molecular species, identical in all their physical and chemical properties, had one other difference: their solutions deviated polarized light in opposite directions. Pasteur hypothesized that these two forms of tartrate corresponded to two different spatial configurations of the atoms within the molecule, two forms which were asymmetrical in themselves, but symmetrical with respect to one another, just as two hands are. This was a revolutionary hypothesis: two molecules, containing the same atoms, linked to one another by the same bonds, could nevertheless differ in the spatial arrangement of these atoms.

This hypothesis, which would not be definitively confirmed until 30 years later with the establishment of the principle of carbon asymmetry, laid the foundation for stereochemistry. We can only fully appreciate the far-reaching consequences of this concept by recalling the fact that, as Pasteur himself sensed and as molecular biology would demonstrate many years later, all interactions between biological molecules, and hence all life processes, result from the precise three-dimensional arrangement of the atoms within these molecules.

Upon observing that all compounds whose solutions deviated polarized light arose from plant and animal sources, Pasteur began to suspect that asymmetry was a sign of life. With this idea in mind, he began his studies on fermentation in 1854. At the time, the question of fermentation was the subject of utmost confusion. While in some cases the participation of microorganisms in such transformations of organic matter was recognized, in general their role was completely misunderstood. Justus Liebig, the renowned German chemist, who was a contemporary and adversary of Pasteur, had stated, "Yeast from the malt ... [transfers] its own state of decomposition to that which is around it. The movement that disturbs the balance imprinted in its own elements also communicates with other elements of bodies in contact with it."

Needless to say, Pasteur could not accept such a confused explanation. The observation that optically active products appeared during the process of fermentation lead him to propose 
an entirely different and much more accurate hypothesis. To Pasteur, "fermentation, far from being a lifeless phenomenon, is a living process. ... All phenomena of fermentation correlate with the development of mycodermic cells and plants which I have prepared and studied in an isolated and pure state." Indeed, his publication of 1857, "Note on lactic fermentation", can be considered to be the birth certificate of microbiology: "The purity of a ferment, its homogeneity, its individual development with the help of a nutrient perfectly adapted to its individual nature, this is one of the essential conditions for obtaining high-quality fermentation."

Fermentation and putrefaction were often perceived as spontaneous phenomena. But what was Pasteur to make of the ferments and yeasts which he felt were essential in the unfolding of those processes? Where did they come from? Did they appear spontaneously in the media, or did they come from elsewhere? Pasteur could not escape this debate, a debate stemming from ancient beliefs concerning the spontaneous generation of bees, frogs, mice, etc., but which had taken on new strength toward the end of the 17th century following the discovery of "animalcules" by Anton van Leeuwenhoek, the Dutch inventor of the microscope. In the 18th century, these microscopic organisms were considered to arise by "spontaneous generation" by scholars such as England's John Needham and France's Buffon, who debated this issue with other scientists such as the Italian Abbot Spallanzani.

Pasteur kept an open mind on this question. In 1859 , in a letter to the man who was to become his most ardent adversary on the subject, Felix Archimede Pouchet, he wrote that the question of spontaneous generation was "entirely open and still awaiting proof", and that all of this was "unknown and warranted experimentation". Pasteur then took up his own invitation. Using extremely painstaking experimental methods, he demonstrated that the appearance of microorganisms in a presterilized medium was always explained by germs coming from the outside. Thus, he succeeded in discrediting any experimental basis for the theory of spontaneous generation.

Pasteur's research on fermentation and on so-called spontaneous generation inevitably led him to the study of infectious diseases. After demonstrating that when environmental yeasts are prevented from being deposited on grapes, the grape juice will not ferment, he wrote, "By analogy, is it unreasonable to hope that the day will come when easily administered preventive measures will be able to stop the scourges which terrify and decimate populations, such as yellow fever and the bubonic plague?" While Pasteur did not attempt to deal with either of those diseases he contributed to the identification of the anthrax agent, staphyloccus, streptococcus, and the septic vibrion.

What was uniquely important about the entire body of Pasteur's work is that it laid the groundwork upon which the microbial theory of disease was built. He demonstrated how to culture bacteria, and later, for rabies, he set forth the premises for culture of viruses on animal tissues. He had always been intrigued by the mechanisms by which pathogenic microbes caused profound disturbances in the physiology of the infected organism. He demonstrated how pathogens were able to spread throughout animal and human populations, thereby laying the foundation for infectious epidemiology and defining the basic rules of hygiene.

Finally, he outlined the overall principles of vaccination and was one of the founders of immunology. The concept of acquired immunity dates from ancient times. Indeed, Thucydides reported that those who were cured of the plague no longer ran the risk of falling victim to the disease. The first vaccine was developed by the English physician, Edward Jenner, who at the end of the 18th century discovered that humans could be protected against smallpox by inoculating them with pustules taken from bovines afflicted with a similar but relatively benign disease called cowpox. Although of crucial importance, since it led to complete eradication of smallpox in 1977, this empirical discovery could not be generalized to other diseases, for it lacked a conceptual basis.

Pasteur's fundamental discovery in this field dates back to 1879 and the disease known as fowl cholera, which was rampant in chicken coops at that time. The disease is due to bacteria that now bear the name "Pasteurella". When inoculated into a hen, several drops of a culture of this bacteria were sufficient to kill the animal. But Pasteur noted, partly by chance it would seem, that hens inoculated with an old culture not only did not die but were protected against a later inoculation with a virulent culture. The principle of vaccination using attenuated pathogens was thus discovered.

From then on, Pasteur continued to apply this principle to other diseases. His first great success, both at a scientific level and in terms of 
public opinion (today we would refer to it as a "mass media happening"), was the vaccination against anthrax. The famous public experiment held in Pouilly-le-Fort in 1881, where 24 vaccinated sheep survived an injection of the anthrax bacillus while 24 nonvaccinated sheep died, had extraordinary repercussions, convincing the public of the validity of Pasteur's work.

The few remaining skeptics rallied around Pasteur after he achieved his final victory, that of human vaccination against rabies. The problem was complicated from the very outset, as the rabies microbe was invisible (we now know that it is a virus rather than a bacterium) and did not multiply in any culture medium. But the stakes were high, for although the disease was relatively rare in France, it had always fascinated the popular imagination, conjuring up fear and mystery. For Pasteur, conquering rabies would consolidate the final victory of his theories.

The success of the vaccination of Joseph
Meister on July 6, 1885, and of the shepherd boy Jean-Baptiste Jupille in October of that same year, followed by hundreds of other bitevictims from all over the world, brought glory to Pasteur and opened up the era of preventive medicine. On the heels of this success, the Academie des Sciences launched an international fund-raising campaign to build the Institut Pasteur. It was there that Louis Pasteur lived out his last years; it is there that he lies in his final resting place, and it is there, for the last 100 years, that the work he began is being pursued by a myriad of his followers.

The Editors of Molecular Medicine have joined the Institut Pasteur in celebrating "The Year of Louis Pasteur" by dedicating the September 1995 issue of the journal to Pasteur and to his contributions to molecular medicine. We hope that at the end of this year Pasteur's achievements will have become better known and appreciated. 